Mon. Not. R. Astron. Soc. 000, ??-?? (2016)～Printed 2nd June $2016 \quad$ (MN LATEX style file v2.2)

\title{
Discovery of hard phase lags in the pulsed emission of GRO J1744-28
}

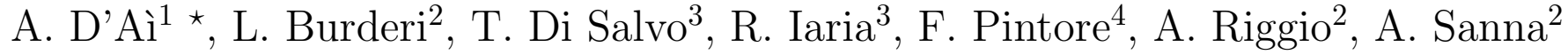 \\ 1 INAF - IASF Palermo, via Ugo La Malfa 153, 90146, Palermo, Italy \\ 2 Dipartimento di Fisica, Università degli Studi di Cagliari, SP Monserrato-Sestu KM 0.7, 09042, Monserrato, Italy \\ 3 Dipartimento di Fisica e Chimica, Università degli Studi di Palermo, via Archirafi 36, 90123, Palermo, Italy \\ 4 INAF - IASF Milano, Via E. Bassini 15, I-20133 Milano, Italy
}

2nd June 2016

\begin{abstract}
We report on the discovery and energy dependence of hard phase lags in the $2.14 \mathrm{~Hz}$ pulsed profiles of GRO J1744-28. We used data from XMM-Newton and NuSTAR. We were able to well constrain the lag spectrum with respect to the softest $(0.3-2.3$ $\mathrm{keV}$ ) band: the delay shows increasing lag values reaching a maximum delay of $\sim 12 \mathrm{~ms}$, between 6 and $6.4 \mathrm{keV}$. After this maximum, the value of the hard lag drops to $\sim 7 \mathrm{~ms}$, followed by a recovery to a plateau at $\sim 9 \mathrm{~ms}$ for energies above $8 \mathrm{keV}$. NuST AR data confirm this trend up to $30 \mathrm{keV}$, but the measurements are statistically poorer, and therefore, less constraining. The lag-energy pattern up to the discontinuity is well described by a logarithmic function. Assuming this is due to a Compton reverberation mechanism, we derive a size for the Compton cloud $R_{\mathrm{cc}} \sim 120 R_{\mathrm{g}}$, consistent with previous estimates on the magnetospheric radius. In this scenario, the sharp discontinuity at $\sim 6.5 \mathrm{keV}$ appears difficult to interpret and suggests the possible influence of the reflected component in this energy range. We therefore propose the possible coexistence of both Compton and disk reverberation to explain the scale of the lags and its energy dependence.
\end{abstract}

Key words: line: identification - line: formation - stars: individual (GRO J1744-28) - X-rays: binaries — X-rays: general

\section{INTRODUCTION}

The transient, X-ray binary pulsar GRO J1744-28, also known as the Bursting Pulsar due to the presence of recurrent Type-II bursts, went in outburst in 2014 mid-January, reached a peak luminosity in mid-March, and returned to quiescence at the end of April. During this outburst, it was observed by many X-ray observatories: results from spectral analysis done with XMM-Newton and INTEG$R A L$ are reported in D'Aì et al. (2015), with Chandra in Degenaar et al. (2014) and with Chandra and NuSTAR in Younes et al. (2015). The pulsar $\left(P_{\text {spin }}=467 \mathrm{~ms}\right)$ orbits in a nearly circular path (eccentricity $<1.1 \times 10^{-3}$ ) with a period of $11.8337 \mathrm{~d}$, and a projected semi-major axis of 2.6324 lt-s. The mass function $\left(1.3638 \times 10^{-4} \mathrm{M}_{\odot}\right)$ indicate an evolved low-mass $\left(M_{\odot} \sim 0.2 M_{\odot}\right)$ companion star and a nearly faceon inclination angle (Finger et al. 1996).

* antonino.dai@icf.inaf.it
The X-ray pulsed profile can be satisfactorily decomposed into the sum of two sinusoids, where the first harmonic accounts for about 3-8\% of the overall harmonic content (Finger et al. 1996; D'Aì et al. 2015; Younes et al. 2015). The profile's shape is energy-dependent; the pulse amplitude is generally positively correlated with energy, with an amplitude fraction of $\sim 5 \%$ for energies below $2 \mathrm{keV}$, increasing to more than $20 \%$ at $\sim 10 \mathrm{keV}$. The amplitude fraction has, however, a drop in the energy range corresponding to the iron fluorescence emission, because of the relative higher contribution to the observed flux from incoherent emission from the reflection component (D'Aì et al. 2015).

Calculations on the long-term evolution of the system that account for the present-day orbital parameters (Rappaport \& Joss 1997), measures of the neutron star (NS) spin-up rate (Finger et al. 1996), and evidence for a propeller state at the end of its 1996 outburst (Cui 1997) indicated an intermediate NS dipole field of a few $10^{11} \mathrm{G}$. Detection of a cyclotron resonance feature between 4 and 
$5 \mathrm{keV}$ in the X-ray spectrum confirmed such expectations (D'Aì et al. 2015; Doroshenko et al. 2015) and firmly established GRO J1744-28 as a peculiar system in-between the class of the highly magnetised $\left(B \gtrsim 10^{12} \mathrm{G}\right)$, young, X-ray pulsars and the class of old NSs with low-mass companions, i.e. accreting ms X-ray pulsars (AMXPs) and not-pulsating accreting NSs, owing a presumed low magnetic dipole field, $\left(\mathrm{B} \lesssim 10^{9} \mathrm{G}\right)$.

In this Letter we focus on the relation between the pulse phase and energy during the persistent (non-bursting) emission of the source. We show the existence of energydependent hard phase lags, we study its dependence and propose a physical scenario using a mix of Compton and disk reverberation.

\section{OBSERVATION, DATA REDUCTION AND ANALYSIS}

XMM-Newton observed GRO J1744-28 on 2014 March 6. Details on this observation are reported in D'Aì et al. (2015). For this work we use only data from the EPIC/pn instrument, screening from type-I bursts and considering only the persistent emission. We applied standard filtering criteria and included events only in the RAWX[28:46] columns. The light curve exposure is $64.1 \mathrm{ks}$ and the averaged rate is $\sim 660 \mathrm{cps}$. Events were barycentred with respect to the Barycentric Dynamical Time (TDB) using the barycen tool with $\mathrm{RA}=266.137792$ and Dec $=-28.740803$ (Gosling et al. 2007). To correct the pulse arrival times for the orbital motion of the pulsar, we adopted the following orbital parameters (Fermi/GBM pulsar project1 $): P_{\text {orb }}=11.836397 \mathrm{~d}$, a $\sin (i)=2.637 \mathrm{lt}-\mathrm{s}$, and $\mathrm{T}_{\pi / 2}=56695.6988 \mathrm{MJD}$. We then used an epoch-folding search (efsearch in XRONOS) to find the averaged pulse period during the persistent emission. The $\chi^{2}$ highest peak is found in correspondence with a period of 0.4670450 (1) s. We selected 20 energy bands in the EPIC/pn spectrum, keeping the number of events approximately equal in each selection. The softest band comprises the $0.3-2.3 \mathrm{keV}$ range, while the hardest band the 9.18-12 keV range. For each energy-filtered event file, we obtained the corresponding folded profile using as folding period $P_{\text {spin }}=0.4670450 \mathrm{~s}$ and a phase-bin time of $\sim 0.5 \mathrm{~ms}$. We fitted the pulsed profiles using the best-fitting function:

$f(x)=C+A_{1} \sin \left(2 \pi\left(x-\Delta \phi_{1}\right)\right)+A_{2} \sin \left(4 \pi\left(x-\Delta \phi_{2}\right)\right)(1)$

where C, $A_{1}, A_{2}, \Delta \phi_{1}$, and $\Delta \phi_{2}$ are the averaged profile count rate, the sinusoidal semi-amplitudes of the fundamental and first overtone and their phase differences with respect to the best-fitting phase of the first interval, respectively.

We obtained a satisfactory description of all the profiles, with an averaged reduced $\chi^{2}$ for the 20 fits of 1.00 (929 d.o.f. per fitted profile) and a standard deviation of 0.05 . We show in Fig. 1 the results for the $\Delta \phi_{1}$ (in units of millisecond time difference with respect to the first energy selected interval). Because we report the phases with respect to the best-fitting phase of the first interval, there is a systematic error of $\sim 1$

1 http://gammaray.msfc.nasa.gov/gbm/science/pulsars/ lightcurves/groj1744.html

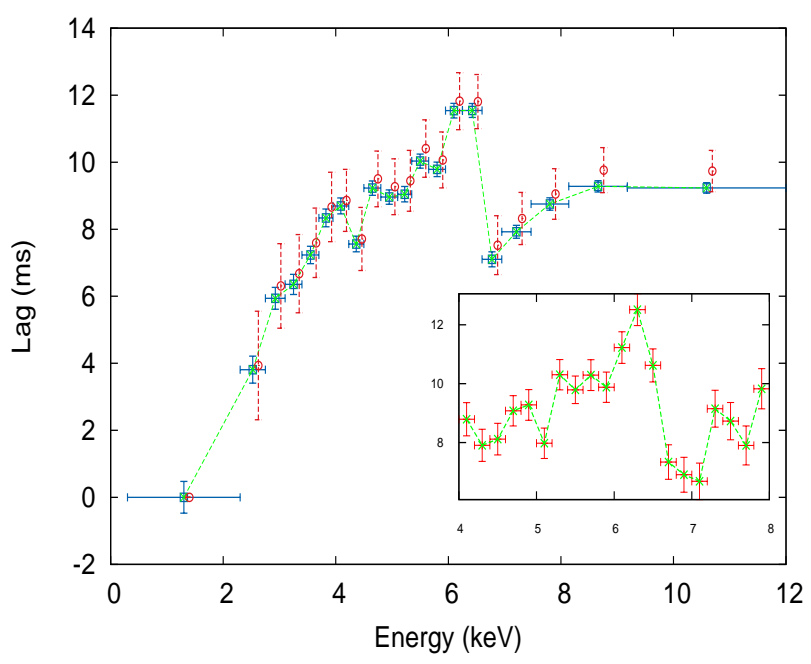

Figure 1. Phase lag dependence with respect to the softest energy band $(E<2.3 \mathrm{keV})$. Blue open squares: data from leastsquared fits to the folded profile. Red open circles: data from cross-correlation technique, shifted of $0.1 \mathrm{keV}$ in energy for clarity's sake. The inset shows the phase dependence in the 4.0-8.0 $\mathrm{keV}$ range, adopting a finer grid (step of $0.2 \mathrm{keV}$ width) for the folded profiles with the least-squared fits.

$\mathrm{ms}$ in the $y$-axis, while error-bars in the plot show only the statistical error at $68 \%$ confidence level. There is a clear hard-phase lag that from the softest band rises up to $12 \mathrm{~ms}$ for energies $\sim 6 \mathrm{keV}$. Above $6.5 \mathrm{keV}$ the lag sharply drops, progressively recovering to form a plateau for energies above $\sim 8 \mathrm{keV}$. Adopting also a different energy binning (with a constant energy width of $200 \mathrm{eV}$ ) and repeating the steps of our analysis, we ensured that the drop is not an artefact of the binning choice (see the inset in Fig. 1).

We applied a second procedure to check the robustness of the trend, using the cross-correlation technique. We obtained the cross-correlation function ( $\mathrm{CCF}$ ) of each energyselected profile with respect to the first folded profile. The resulting CCF had a clear bell-shaped function; we obtained the centre of this function through a Gaussian fit in a restricted interval around the CCF peak. To estimate the error on the data, we generated for each profile 1000 simulated profiles based on the same statistics of the original profile. We found again the set of best-fitting centre values and we choose to associate as error the standard deviation of this sample. The final result is shown in Fig. 1 (red open circles points) together with the results from the first method to allow for a quick comparison. The trend is fully compatible with our first procedure, the best-fitting lags are slightly above the corresponding best-fitting values of the least-squared lags (still well within the error bars) but the associated errors are systematically larger due to the greater uncertainty in estimating the peak of the cross-correlation function.

We also analysed two NuSTAR observations of GRO J1744-28 performed on 2014 February 15 (OBS.Id 80002017002 , net exposure $24 \mathrm{ks}$, rate $185 \mathrm{cps}$ ) and on 2014 March 3 (Obs.ID 80002017004, net exposure $7.1 \mathrm{ks}$, rate 570 cps). We selected a circular region centred on the source coordinates of 100 " radius for both observations, and applied the same procedure for the analysis of the $\mathrm{EPIC} / \mathrm{pn}$ data, 


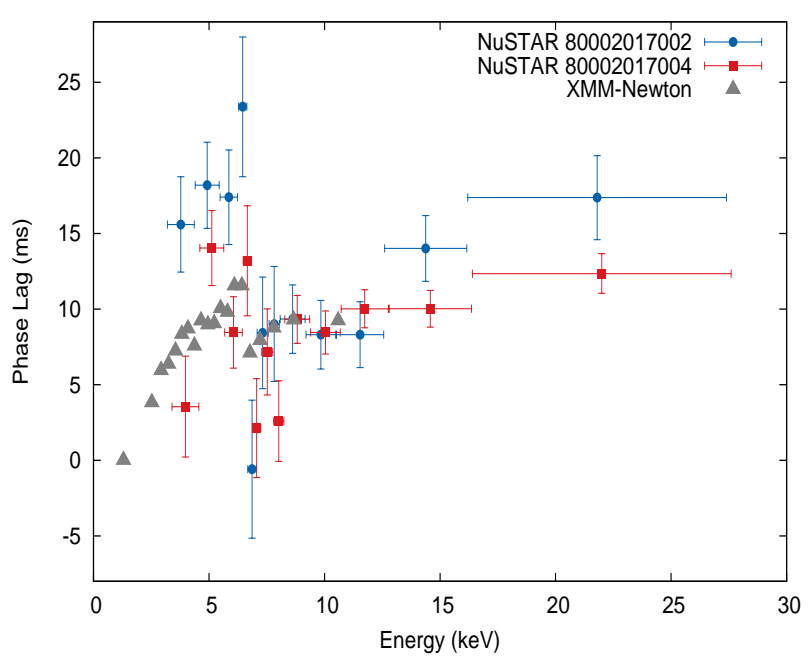

Figure 2. Phase-lags for the two $N u S T A R$ observations: in blue and red data from the 2014-02-15 and 2014-03-03 observations, respectively. We applied a small shift of $0.1 \mathrm{keV}$ in energy for clarity's sake. Grey filled triangles show the XMM-Newton data points.

selecting 12 energy bands. To improve the statistics, we combined the light curves of the two Photon Counting Detector Modules (FPMA and FPMB) to derive a single folded profile. We obtained as best folding periods $0.4670460 \mathrm{~s}$ and $0.4670453 \mathrm{~s}$, for the first and second observation, respectively.

In Fig. 2 we show the phase-lag dependence according to the least-square method. To allow a direct comparison with the XMM-Newton data, we over-plotted them (grey triangles), setting the reference phase of the phase-lag at $\sim 8.8 \mathrm{keV}$ channel to be equal to the value found for the EPIC/pn data-set. The NuST AR data show in both observations qualitatively a trend similar to the one shown by EPIC/pn data. Quantitatively, the lag values are much less constrained and they do not allow us to draw firm conclusions about possible differences in the trends derived for the two observations, although there is a hint for greater lags in the NuSTAR observation of February, when the source's luminosity was about a third of the March observation.

We conservatively limit the discussion to the much more clearly constrained dependence outlined by the EPIC/pn data.

\section{DISCUSSION}

Energy-dependent soft phase-lags in accreting X-ray pulsars have been reported for sources belonging to the class of the millisecond pulsars (Cui et al. 1998; Galloway et al. 2002, 2005; Ibragimov et al. 2011; Falanga et al. 2012). Recently Mivasaka et al. (2013) reported an energy dependent hard phase-lag for the slowly spinning $\left(P_{\text {spin }}=12.29 \mathrm{~s}\right)$, X-ray pulsar GS 0834-430, and an energy-modulated phase-lag has been reported for $4 \mathrm{U} 0115+63$ (Ferrigno et al. 2009). The fractional delay for AMXPs is of the order of $5 \%$ and soft photons lag harder ones, whereas the hard phase lag in GS 0834-430 shows a monotonic rise with energy attaining a $30 \%$ of the pulse period above $50 \mathrm{keV}$. GRO J1744-28 energy dependent phase lag more closely resembles the behaviour of GS $0834-430$, but its fractional delay ( $2.6 \%$ of the pulse period) is significantly lower.

Different interpretations have been widely discussed in literature about the origin and the possible diagnostic tools offered by such measurements (Poutanen 2001). A straight geometric interpretation of the lags due to the different geometrical paths travelled by photons of different energy bands are advocated in the case of some accreting X-ray binaries and AGNs, where part of the hard photons are reflected by the surface of the accretion disk. The lag is thus a measure of the reverberation distance between the primary source of the incident photons and the averaged radius in the disk where reflection actually occurs; Fourier-resolved time-lags have provided an additional method to connect the intrinsic variability of the irradiating flux with the scattered/reflected component (Uttlev et al. 2014).

The non-monotonic lag-energy spectrum of Fig. 1 clearly shows a smooth rising trend and a discontinuity in the iron range, where a broad emission line is present in the energy spectrum (D'Aì et al. 2015). We shall therefore explore a scenario where a combination of Compton and disk reverberation might both operate to produce the observed lag dependence. We start making some preliminary considerations on the size of its accretion disk. Assuming a NS mass of $1.69 M_{\odot}$, a companion's mass of $0.24 M_{\odot}$ (Rappaport \& Joss 1997) and the binary period of 11.83 days (Finger et al. 1996), we find that the pulsar Roche lobe radius is $\sim 43 \mathrm{lt}-\mathrm{s}$, and the circularization radius $\sim 4$ lt-s (Eq. 4.6 and 4.21 in Frank et al. 2002), so that the outer disk radius, which should lie in between these two values, would easily accommodate the overall variation scale of the observed lags. The inner disk radius, if truncated at the magnetospheric radius, should instead be of the order of 1 lt-ms (D'Aì et al. 2015).

The reflection component in the energy spectrum of GRO J1744-28 gives its strongest contribution between 6.6-6.8 keV, because of the presence of a moderately broadened emission line from highly ionised He-like iron ions (Degenaar et al. 2014; Younes et al. 2015; D'Aì et al. 2015). The ratio $R$ of the reflected and the continuum fluxes is almost constant in the $2-6 \mathrm{keV}$ range at $\sim 5 \%$ and rapidly rises up to $\sim 15 \%$ at the iron peak (see lower panel of Fig 3). Presence of this emission is also noticeable from the clear drop in the pulsed emission amplitude at this energy, because the reflected component, coming from at least a distance of $1 \mathrm{lt}-\mathrm{ms}$, has lost coherence with respect to the non-incident continuum (see upper panel of Fig. 3 and also Fig. 12 in D'Aì et al. 2015). It is therefore plausible that a distortion of the pulsed profile can be caused by some reverberation on the disk, if the reflected emission is seen pulsating (at least partially), but with a phase difference with respect to the direct emission. The energy-dependent sign of the lags from the iron emission line depends on the distance where photons are actually emitted, so that they track the complex, broadened, line shape, with the extended red wing and the Doppler boosted blue peak coming from the inner radii (shorter lags), and the line core, close at the rest-frame energy, produced by the more distant radii (longer lags), where relativistic and dynamical effects are strongly damped (Campana \& Stella 1995). Qualitatively, the lag spectrum, shown in the middle panel of Fig. 3. re- 


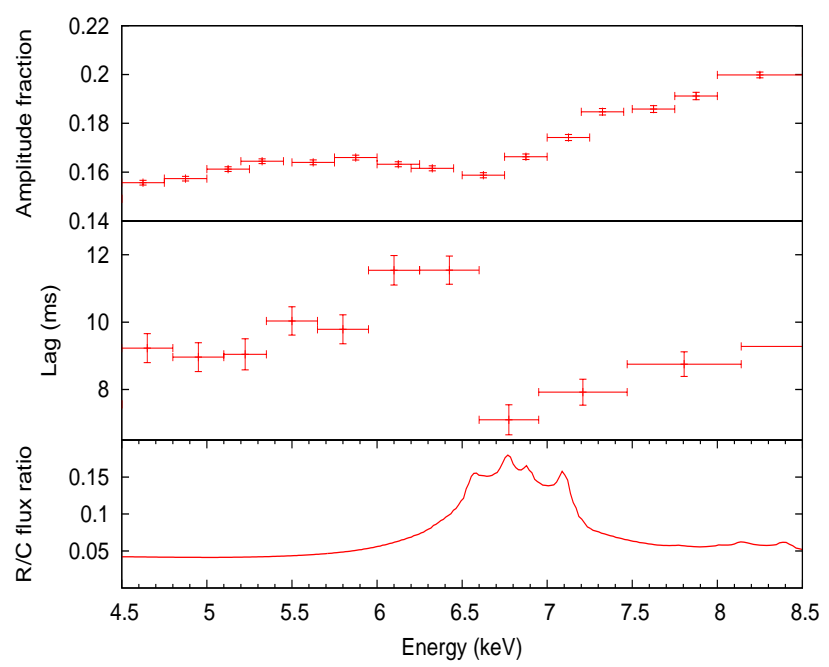

Figure 3. Amplitude fraction (upper panel) and phase-delay (middle panel) of the fundamental harmonic as function of energy in the 4.5-8.5 keV energy range. Ratio of the fluxes from the reflected and the irradiating continuum (lower panel), according to the spectral decomposition in D'Aì et al. (2015).

produces these features: the overall scale of the lag in this energy range is $3-5 \mathrm{~ms}$, consistent with the dimensions of the inner parts of the accretion disk. When the line emission drops, the lag values at the extreme wings of the line are consistent. We also note that the highest lag value is at $\sim 6.4$ $\mathrm{keV}$, where Younes et al. (2015) detected possible emission from neutral/lowly ionised iron, which may hint also to a contribution from a neutral and more distant reflector.

When the energy-lag spectrum is considered below 6.5 $\mathrm{keV}$, the lag values rise almost monotonically. As a possible mechanism able to produce this monotonic part of the lag trend, we consider the Compton reverberation scenario (Payne 1980; Guilbert et al. 1982). In this case, energetic, relatively hot electrons up-scatter colder photons produced by bremsstrahlung, synchrotron processes or emerging from the thermalised mould at the base of the accretion column. If the delay is produced because hard photons spend more time in the Comptonizing cloud due to repetitive scattering, we can derive from the dependence of the delay on the photon energy an estimate on the size of the cloud, assuming reasonable values for the electron temperature and optical depth, together with the very simplifying assumption of an uniformly dense and static corona (but see Hua et al. 1997, for a corona with a density gradient).

Because of the clear discontinuity at $\sim 6.6 \mathrm{keV}$, we first fitted the phase delays obtained from the least-squared fits (Fig. 1. blue open squares), in the restricted $0.3-6.6 \mathrm{keV}$ energy range, assuming a logarithmic dependence function, according to the formula (Lightman et al. 1978):

$T_{\text {lag }}=\frac{R_{c c}}{c(1+\tau)} \frac{\ln \left(E_{h} / E_{s}\right)}{\ln (1+4 \Theta(1+4 \Theta))}$

where $\Theta=k T_{e} / m c^{2}, \tau$ and $R_{c c}$ are the optical depth and the Compton cloud size, respectively. $E_{h}$ and $E_{s}$ are the hard and soft photons energy $\left(E_{s}=1.3 \mathrm{keV}\right.$ is a fixed parameter, being the central value of the first energy band), and $c$ is the speed of light.

The logarithmic function provides a satisfactory de-

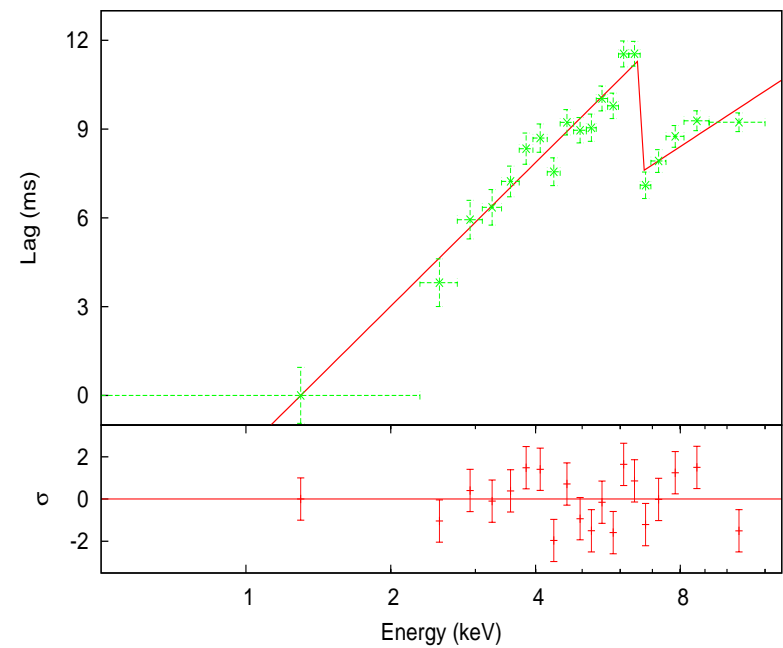

Figure 4. Best-fitting piece-wise function (Eq. 2] with a break at $6.6 \mathrm{keV}$ ) of the EPIC/pn data (Fig. 1) and residuals in units of $\sigma$ (lower panel).

scription of the delays up to $6.6 \mathrm{keV}$ (reduced $\chi^{2}$ of 1.3 with 15 degrees of freedom), with a best-fitting value of the constant before the logarithm of $7.0 \pm 0.1 \mathrm{~ms}$.

There is small uncertainty for the cut-off energy of the spectrum. Adopting a variety of models and also from independent observations and analysis of the broadband spectrum of GRO J1744-28 (D'Aì et al. 2015; Younes et al. 2015), the cut-off energy of the spectrum results $\sim 7 \mathrm{keV}$, so that $\Theta \sim 1.3 \times 10^{-2}$. The optical depth is difficult to constrain from the simple observation of the slope of the spectrum, because the exact value depends on the assumptions about the geometry of the Comptonization region and on a variety of concomitant physical processes that are superimposed on the observed spectrum (i.e. black-body emission from the NS cap, synchrotron emission, and the ratio of bulk versus thermal Comptonization). Moreover, the scattering cross-section depends on the direction of the photon with respect to the magnetic field lines, allowing radiation to escape more freely through the walls of the accretion column. We consider a possible lower limit of $\tau=1$ (see Sect. 5.2 in Becker \& Wolff 2007) and estimate the region size $R_{c c}$ to be of the order of $\sim 0.8$ ms-lt $(240 \mathrm{~km})$, that would be consistent with the estimates on the magnetosphere size as derived by the measure of the inferred NS magnetic dipole and the dynamical and relativistic broadening of the fluorescence lines of the reflection component (Degenaar et al. 2014; D'Aì et al. 2015).

We note that after the discontinuity the trend suggests a return to a logarithmic dependence as confirmed by the extended $N u S T A R$ coverage, but with a different multiplicative constant (Fig. 1). In fact, adopting a piecewise function, with $\mathrm{f}(\mathrm{x})=k_{1} \log \left(E_{h} / E s\right)$ for $E<6.6 \mathrm{keV}$ and $\mathrm{f}(\mathrm{x})=k_{2} \log \left(E_{h} / E s\right)$ for $E>6.6 \mathrm{keV}$, we obtained a general satisfactory fit $\left(\chi_{\text {red }}^{2}=1.47\right.$ for 18 dof $)$ as shown in Fig. 4. The best-fitting constant of this second logarithmic function $\left(k_{2}=4.5 \mathrm{~ms}\right)$ would point to physical characteristics similar to the ones of the previously discussed region. In conclusion, if the whole lag spectrum is interpreted only as due to Compton reverberation, we would need an unreal- 
istic break in the iron range, requiring two Comptonizing separate regions but with similar physical parameters. On the contrary, it appears more likely that the sharp discontinuity is determined by the over-imposed effect of the disk reverberation. In this case, it remains to explain why at the iron core energy the over-imposed hard lag, is not above the extrapolated best-fitting function that fits the softer 1-6.4 $\mathrm{keV}$ lag range of Fig. 4 We argue that if the continuum emission is emitted in a fan-beam geometry, as possibly envisaged for pulsars accreting at very high $\dot{M}$ Basko \& Sunvaev 1976), and the GRO J1744-28 is a system seen at low inclination angle, with the magnetic columns almost parallel to our line-of-sight, the optical paths of the continuum photons hitting the disk and directed along our line-of-sight could be different. Part of the continuum emission that impinges on the disk escapes laterally from the base of the shock region, whereas the Comptonizing cloud that produces the continuous phase-shift of the pulsed emission is along our line-of-sight. Although the details of this combined process could be much more complex, we note that at least qualitatively this sketched scenario could explain both the scale of the observed lags and its trend. We note, that, besides the Compton reverberation, there are other ways to produce a logarithmic lag-energy dependence: e.g. as suggested by Kotov et al. (2001) if the slope of the power-law depended on the distance, with harder indices produced in the inner regions, and softer in the outer regions, a logarithmic lag-energy spectrum is naturally produced. Moreover, this mechanism coupled with a reflecting disk, would produce a complex lag-spectrum, with a dimple (also defined by Kotov et al. 2001, anti-lags, as they anticipate the softer band) at the energies of the fluorescent iron line very similarly to what observed in the GRO J1744-28.

Finally, we also mention the possibility that the lag discontinuity might be caused by an abrupt change in the energydependent scattering cross-section at the cyclotron resonant energy (as suggested for the accreting pulsar 4U 0115+63 by Ferrigno et al. 2009), though the results from spectral analysis in D'Aì et al. (2015) indicated the presence of a moderately broad absorption feature at lower energy $\sim 4.7$ $\mathrm{keV}$.

\section{ACKNOWLEDGEMENTS}

The High-Energy Astrophysics Group of Palermo acknowledges support from the Fondo Finalizzato alla Ricerca (FFR) 2012/13, project N. 2012-ATE-0390, founded by the University of Palermo. A. R. gratefully acknowledges the Sardinia Regional Government for the financial support (P. O. R. Sardegna F.S.E. Operational Programme of the Autonomous Region of Sardinia, European Social Fund 2007-2013 - Axis IV Human Resources, Objective 1.3, Line of Activity 1.3.1).

The Palermo and Cagliari University groups acknowledge financial contribution from the agreement ASI-INAF I/037/12/0.

\section{References}

Basko M. M., Sunyaev R. A., 1976, MNRAS, 175, 395

Becker P. A., Wolff M. T., 2007, ApJ, 654, 435

Campana S., Stella L., 1995, MNRAS, 272, 585

Cui W., 1997, ApJL, 482, L163

Cui W., Morgan E. H., Titarchuk L. G., 1998, ApJL, 504, L27
D'Aì A., Di Salvo T., Iaria R., García J. A., Sanna A., Pintore F., Riggio A., Burderi L., Bozzo E., Dauser T., Matranga M., Galiano C. G., Robba N. R., 2015, ArXiv e-prints

Degenaar N., Miller J. M., Harrison F. A., Kennea J. A., Kouveliotou C., Younes G., 2014, ApJL, 796, L9

Doroshenko R., Santangelo A., Doroshenko V., Suleimanov V., Piraino S., 2015, ArXiv e-prints

Falanga M., Kuiper L., Poutanen J., Galloway D. K., Bozzo E., Goldwurm A., Hermsen W., Stella L., 2012, A\&A, 545, A26

Ferrigno C., Becker P. A., Segreto A., Mineo T., Santangelo A., 2009, A\&A, 498, 825

Finger M. H., Koh D. T., Nelson R. W., Prince T. A., Vaughan B. A., Wilson R. B., 1996, Nature, 381, 291

Frank J., King A., Raine D. J., 2002, Accretion Power in Astrophysics: Third Edition. Accretion Power in Astrophysics, by Juhan Frank and Andrew King and Derek Raine, pp. 398. ISBN 0521620538. Cambridge, UK: Cambridge University Press, February 2002.

Galloway D. K., Chakrabarty D., Morgan E. H., Remillard R. A., 2002, ApJL, 576, L137

Galloway D. K., Markwardt C. B., Morgan E. H., Chakrabarty D., Strohmayer T. E., 2005, ApJL, 622, L45

Gosling A. J., Bandyopadhyay R. M., Miller-Jones J. C. A., Farrell S. A., 2007, MNRAS, 380, 1511

Guilbert P. W., Fabian A. C., Ross R. R., 1982, MNRAS, 199, 763

Hua X.-M., Kazanas D., Titarchuk L., 1997, ApJL, 482, L57

Ibragimov A., Kajava J. J. E., Poutanen J., 2011, MNRAS, 415, 1864

Kotov O., Churazov E., Gilfanov M., 2001, MNRAS, 327, 799

Lightman A. P., Giacconi R., Tananbaum H., 1978, ApJ, 224, 375

Miyasaka H., Bachetti M., Harrison F. A., 2013, ApJ, 775, 65

Payne D. G., 1980, ApJ, 237, 951

Poutanen J., 2001, Advances in Space Research, 28, 267

Rappaport S., Joss P. C., 1997, ApJ, 486, 435

Uttley P., Cackett E. M., Fabian A. C., Kara E., Wilkins D. R., 2014, A\&ARv, 22, 72

Younes G., Kouveliotou C., Grefenstette B. W., 2015, ApJ, 804, 43 